\title{
Quantum Optics in Dispersive and Absorptive Media
}

\author{
Akira Shimizu円, Teruaki Okushima and Kazuki Koshino \\ Institute of Physics, University of Tokyo, 3-8-1 Komaba, Meguro-ku, Tokyo 153, Japan
}

\begin{abstract}
Using microscopic models in which both photons and excitons are treated as microscopic degrees of freedom, we discuss polaritons of two cases: One is the case when excitonic parameters are time dependent. The time dependence causes creation of polaritons from a "false vacuum." It is shown that both the creation sepctra and the creation efficiency are much different from the results of the previous studies. The other is polaritons in absorptive and inhomogeneous cavities. A polariton in such a system cannot be viewed as a back and forth oscillation between a photon state and an exciton state.
\end{abstract}

Keywords: Polariton, Dynamical Casimir effect, Cavity Quantum Electrodynamics, Photon, exciton

\section{Introduction}

Optical phenomena in condensed matter are usually discussed in the case when the optical field can be treated as a classical field. Recently, however, quantum optical phenomena in condensed matter, in which the optical field exhibits its quantum natures in condensed matter, have been attracting much attention. A simple theoretical framework, which is widely used in the literature, for the analysis of such phenomena is to somehow quantize the macroscopic electromagnetic (EM) fields in the "macroscopic Maxwell equations" (Maxwell equations in matter) [1]. The simple theory treats the matter as an effective medium, whose properties are assumed to be fully described by phenomenological parameters such as a dielectric constant and nonlinear susceptibilities. The theory works well for simple problems. However, its validity is not clear when dispersion and absorption is important, when the system is inhomogeneous, when nonlinearities are important, or when the phenomenological parameters are time dependent.

Another way of formulating quantum optics in condensed matter is a microscopic approach. In this case the matter is represented as microscopic polarization fields, and both the EM fields and the polarization fields are quantized. Upon diagonalization of the Hamiltonian of the coupled photon-polarization system, Hopfield [2] obtained "polaritons" as eigenstates of the total system. His theory has been extended by many researchers to study polaritons of various types. For example, polaritons in the case when excitons are subject to dissipation were studied by Huttner, Baumberg and Barnett [3].

In this work, we discuss two cases: One is polaritons in the case when excitonic parameters (the exciton energy and exciton-photon coupling) are time dependent [4. The time dependence causes creation of polaritons from a "false vacuum." This phenomenon is analogous to the dynamical Casimir effect, which is creation of photons by quick movement of mirrors in vacuum [5, 6]. We will present the creation spectra of both the lower- and upper-branch polaritons. The other is polaritons in absorptive and inhomogeneous cavities [7]. We point out that a polariton in such a system cannot be viewed as a back and forth oscillation between a photon state and an exciton state, and a photon state will evolve into a superposition of many, different exciton states, and will not revive. 


\section{Creation of polaritons from a false vacuum of solids}

It is usually assumed in discussions of polaritons that parameters such as the exciton energy or exciton-photon coupling are constant, i.e., independent of time. What happens if the parameters are time dependent? We can show that polaritons will be created even when the initial state is the vacuum. This is a general property of quantum theory: Particles will be created when the Lagrangian (or, almost equivalently, equations of motion) has a time-dependent parameter(s) [5]. This can be understood simply as follows. Suppose that the parameters are constant for $t \leq 0$. For this constant values of parameters we can (in principle) find out the ground state, which is called a "vacuum," in which no polaritons are present. This vacuum is denoted by $\left|\tilde{0}_{1}\right\rangle$. Assume that the parameters vary during $0<t<T$, and again become constant for $t \geq T$. Then for $t \geq T$ we can find out another ground state, which is denoted by $\left|\tilde{0}_{2}\right\rangle$. Under the initial condition that the state vector $|\psi\rangle$ is $\left|\tilde{0}_{1}\right\rangle$ for $t \leq 0$, we may solve the Schrödinger equation [8]. Then we will find that $|\psi\rangle$ at $t \geq T$ differs from $\left|\tilde{0}_{2}\right\rangle$ in general. This means that we have a finite probability of finding particles at $t \geq T$. More particles are normally created for faster variations of parameters (i.e., for shorter $T$ ). This is understood if we think of the limiting case of $T \rightarrow \infty$, for which $|\psi\rangle$ evolves adiabatically from $\left|\tilde{0}_{1}\right\rangle$ at $t \leq 0$ to $\left|\tilde{0}_{2}\right\rangle$ at $t \geq T$, and no particles are created. That is, non-adiabatic (fast) variation is necessary for particle creation.

We here consider the particle creation in condensed matter. Such a phenomenon is known in quantum optics as "spontaneous parametric fluorescence" [9]. The conventional theory of the spontaneous parametric fluorescence is a phenomenological one. That is, it relies on the phenomenological quantization scheme, assuming that dispersion and absorption are absent. It also assumes a phenomenological interaction Hamiltonian of the following form;

$$
H_{\text {int }}=g a_{s}^{\dagger} a_{i}^{\dagger} a_{p}+\text { h.c. },
$$

where $a_{s}, a_{i}$ and $a_{p}$ are annihilation operators of "signal" (s), "idler" (i) and pump photons, respectively, and $g$ is an effective coupling constant which is proportional to the second-order nonlinear susceptibility. Through this interaction, the equations of motion of the signal- and idler-photon fields are modulated at the frequency $\omega_{p}\left(=\omega_{s}+\omega_{i}\right)$ of the pump photon field. That is, the time scale $T$ of the modulation is $T \sim 1 / \omega_{s} \sim 1 / \omega_{i}$. This is a very fast modulation, and the signal and idler photons can be created efficiently enough to be observed experimentally.

Similar situations have been studied by Yablonivitch [10] and by Schwinger [11]. They investigated photon creation in the cases when the dielectric constant $\epsilon$ of material is time dependent. The time dependence may be due to creation of an electron-hole plasma by a short laser pulse [10], or by sudden collapse of a bubble in a liquid [11]. As in the case of the spontaneous parametric fluorescence, the theoretical analyses are phenomenological ones. That is, they rely on the phenomenological quantization scheme, assuming that dispersion and absorption are absent. It was furthermore assumed that $\epsilon$ changes either discontinuously $(T=0)$ [10, 11, 12, or almost adiabatically $(T \sim \infty)$ [10]. The discontinuous change is unphysical, and, as will be shown below, turns out to lead to enormous overestimate of the particle-creation efficiency.

Because of these many assumptions, the previous theories [9, 10, 11] have only a limited range of applicability. For example, they cannot be applied to the case when relevant photon energies $\hbar \omega$ are close to the excitonic energy $\hbar \omega_{x}$, because the dispersion is strong for $\omega \sim \omega_{x}$. However, such a region of the photon energy should be most interesting. Such an interesting 
which is described by the Lagrangian density of the system of the exciton field $\mathbf{X}(\mathbf{r}, t)$, the photon field $\mathbf{A}(\mathbf{r}, t)$, and the longitudinal field $U(\mathbf{r}, t)$ :

$$
\mathcal{L}=\frac{1}{2}\left[\epsilon_{0} E^{2}-\frac{1}{\mu_{0}} B^{2}\right]+\frac{\rho}{2}\left[\frac{\partial X^{2}}{\partial t}-\omega_{x}(t)^{2} X^{2}\right]-\alpha(t)[\mathbf{A} \cdot \dot{\mathbf{X}}+U \nabla \cdot \mathbf{X}]
$$

where the electric and magnetic fields are given by

$$
\mathbf{E}=-\dot{\mathbf{A}}-\nabla U, \quad \mathbf{B}=\nabla \times \mathbf{A} .
$$

This Lagrangian is a generalization to the time-dependent $\omega_{x}$ and $\alpha$ of the model proposed by Huttner et al. [3]. Following them, we apply the standard quantization procedure as follows. We impose the Coulomb gauge condition, so that $\mathbf{A}$ becomes transversal and $U$ can be eliminated. By decomposing $\mathbf{X}$ into the transversal and longitudinal parts,

$$
\mathbf{X}=\mathbf{X}^{\top}+\mathbf{X}^{\|}
$$

we can decompose the Lagrangian $L$ into the transversal and longitudinal parts:

$$
L=\int \mathcal{L} d v=L^{\top}+L^{\|}
$$

Since $\mathbf{A}$ is involved only in $L^{\top}$, we focus on this part. In the Coulomb gauge, it is convenient to quantize the fields in the $\mathbf{k}$ space:

$$
\mathbf{A}(\mathbf{r}, t)=\sum_{\mathbf{k}} \mathbf{A}(\mathbf{k}, t) e^{i \mathbf{k} \cdot \mathbf{r}}, \quad \text { where } \quad \mathbf{A}(\mathbf{k}, t)=\mathbf{A}^{\dagger}(-\mathbf{k}, t)
$$

and similarly for $\mathbf{X}^{\top}$. The fields are further decomposed into two polarizations;

$$
\mathbf{A}(\mathbf{k}, t)=\sum_{\lambda=1,2} A^{\lambda}(\mathbf{k}, t) \mathbf{e}_{\lambda}(\mathbf{k}), \quad \mathbf{k} \cdot \mathbf{e}_{\lambda}(\mathbf{k})=0
$$

and similarly for $\mathbf{X}^{\top}$. By expressing $L^{\top}$ in terms of these components, we can find their conjugate momenta $\Pi^{\lambda}(\mathbf{k}, t)$ and $P^{\lambda}(\mathbf{k}, t)$ by differentiating $L^{\top}$ with respect to $\dot{A}^{\lambda}(\mathbf{k}, t)$ and $\dot{X}^{\lambda}(\mathbf{k}, t)$, respectively. The Hamiltonian $H$ (for the transversal parts) is obtained as

$$
H=\sum_{\mathbf{k} \lambda}\left(\Pi^{\lambda} \dot{A}^{\lambda}+P^{\lambda} \dot{X}^{\lambda}\right)-L^{\top}
$$

Note that this $H$, like $L$, has explicit t dependence, $H=H(t)$, through the $\mathrm{t}$ dependent parameters. The fields are then quantized, in the standard manner, by imposing the equaltime commutation relations;

$$
\left[A^{\lambda}(\mathbf{k}, t), \Pi^{\lambda^{\prime}}\left(\mathbf{k}^{\prime}, t\right)\right]=\left[X^{\lambda}(\mathbf{k}, t), P^{\lambda^{\prime}}\left(\mathbf{k}^{\prime}, t\right)\right]=i \hbar \delta_{\lambda, \lambda^{\prime}} \delta_{\mathbf{k}, \mathbf{k}^{\prime}} .
$$

This leads to the Heisenberg equations of motion;

$$
i \hbar \frac{\partial}{\partial t} A^{\lambda}=\left[A^{\lambda}, H(t)\right], \quad i \hbar \frac{\partial}{\partial t} X^{\lambda}=\left[X^{\lambda}, H(t)\right] .
$$

So far, the calculations are parallel to those of Ref. [3]. The peculiar features of time- 
each $t$. Let $\tilde{a}^{\lambda}(\mathbf{k}, t)$ and $\tilde{b}^{\lambda}(\mathbf{k}, t)$ be the operators which diagonalize the Hamiltonian at time $t$ :

$$
H(t)=\hbar \sum_{\mathbf{k}, \lambda}\left[\tilde{\omega}_{a}(k, t) \tilde{a}^{\lambda \dagger}(\mathbf{k}, t) \tilde{a}^{\lambda}(\mathbf{k}, t)+\tilde{\omega}_{b}(k, t) \tilde{b}^{\lambda \dagger}(\mathbf{k}, t) \tilde{b}^{\lambda}(\mathbf{k}, t)\right]+\text { c-numbers. }
$$

If $\alpha$ and $\omega_{x}$ were constant, $\tilde{a}^{\lambda}$ and $\tilde{b}^{\lambda}$ would have become the usual annihilation operators (times a phase factor, which of course is unimportant) of lower-branch (LB) and upperbranch (UB) polaritons, respectively, with $\tilde{\omega}_{a}$ and $\tilde{\omega}_{b}$ being their eigen-frequencies. However, this is not the case because our $\alpha$ and $\omega_{x}$ depend on $t$. Moreover, it should also be emphasized that the equations,

$$
i \hbar \frac{\partial}{\partial t} \tilde{a}^{\lambda}=\left[\tilde{a}^{\lambda}, H(t)\right], \quad i \hbar \frac{\partial}{\partial t} \tilde{b}^{\lambda}=\left[\tilde{b}^{\lambda}, H(t)\right], \quad \text { (wrong) }
$$

which look like Eq. (10), does not hold for $\tilde{a}^{\lambda}$ and $\tilde{b}^{\lambda}$. For these reasons, the diagonalization of $H$ does not solve the problem.

To solve the problem, we must first specify the initial state vector. For this purpose, we assume that the parameters are constant in the remote past:

$$
\alpha(t) \sim \alpha_{1}, \omega_{x}(t) \sim \omega_{1} \quad \text { as } \quad t \rightarrow-\infty
$$

This ensures that as $t \rightarrow-\infty$ the operators $\tilde{a}^{\lambda}$ and $\tilde{b}^{\lambda}$ and the frequencies $\tilde{\omega}_{a}$ and $\tilde{\omega}_{b}$ approach the annihilation operators $\tilde{a}_{1}^{\lambda}$ and $\tilde{b}_{1}^{\lambda}$ and the eigen frequencies $\tilde{\omega}_{a 1}$ and $\tilde{\omega}_{b 1}$, respectively, of the usual polariton operators, which are for the values of the parameters $\alpha=\alpha_{1}$ and $\omega_{x}=\omega_{1}$. We consider the case when the initial state vector is the vacuum of these polaritons:

$$
|\psi\rangle=\left|\tilde{0}_{1}\right\rangle, \quad \text { where } \quad \tilde{a}_{1}^{\lambda}\left|\tilde{0}_{1}\right\rangle=\tilde{b}_{1}^{\lambda}\left|\tilde{0}_{1}\right\rangle=0
$$

The state vector does not evolve because we are working in the Heisenberg picture. On the other hand, to specify the final states (the vacuum, one-particle states, two-particle states, etc.), we further assume that the parameters are constant also in the remote future:

$$
\alpha(t) \sim \alpha_{2}, \omega_{x}(t) \sim \omega_{2} \text { as } t \rightarrow+\infty
$$

Hence, as $t \rightarrow+\infty$ the operators $\tilde{a}^{\lambda}$ and $\tilde{b}^{\lambda}$ and the frequencies $\tilde{\omega}_{a}$ and $\tilde{\omega}_{b}$ approach the annihilation operators $\tilde{a}_{2}^{\lambda}$ and $\tilde{b}_{2}^{\lambda}$ and the eigen frequencies $\tilde{\omega}_{a 2}$ and $\tilde{\omega}_{b 2}$, respectively, of another polariton operators, which are for the values of the parameters $\alpha=\alpha_{2}$ and $\omega_{x}=\omega_{2}$.

We are interested in the number of created polaritons of each polariton mode in the final state. They are given by

$$
\tilde{n}_{a 2}^{\lambda}(\mathbf{k})=\left\langle\tilde{0}_{1}\left|\tilde{a}_{2}^{\lambda \dagger}(\mathbf{k}) \tilde{a}_{2}^{\lambda}(\mathbf{k})\right| \tilde{0}_{1}\right\rangle, \quad \tilde{n}_{b 2}^{\lambda}(\mathbf{k})=\left\langle\tilde{0}_{1}\left|\tilde{b}_{2}^{\lambda \dagger}(\mathbf{k}) \tilde{b}_{2}^{\lambda}(\mathbf{k})\right| \tilde{0}_{1}\right\rangle,
$$

for each mode of the LB and UB polaritons, respectively. To evaluate these numbers, we must express $\tilde{a}_{2}^{\lambda}$ and $\tilde{b}_{2}^{\lambda}$ in terms of $\tilde{a}_{1}^{\lambda}, \tilde{b}_{1}^{\lambda}$ and their Hermitian conjugates. For our time dependent Lagrangian this relation takes the form of a Bogoliubov transformation, hence $\tilde{n}_{a 2}$ and $\tilde{n}_{b 2}$ become finite [5]. The coefficients of the transformation can be found by solving the Heisenberg equations of motion for the fields, Eq. (10), and inserting the results into the relation between the fields and $\tilde{a}^{\lambda}, \tilde{b}^{\lambda}, \tilde{a}^{\lambda \dagger}$ and $\tilde{b}^{\lambda \dagger}$. 
We have performed a numerical calculation taking the following forms for $\omega_{x}(t)$ and $\alpha(t)$;

$$
\begin{aligned}
& \omega_{x}(t)= \begin{cases}\omega_{1} & (t \leq 0) \\
\omega_{1}-\frac{\omega_{1}-\omega_{2}}{2 \pi}\left[\frac{2 \pi t}{T}-\sin \left(\frac{2 \pi t}{T}\right)\right] & (0<t<T) \\
\omega_{2} & (t \geq T)\end{cases} \\
& \alpha(t)= \begin{cases}\alpha_{1} & (t \leq 0) \\
\alpha_{1}-\frac{\alpha_{1}-\alpha_{2}}{2 \pi}\left[\frac{2 \pi t}{T}-\sin \left(\frac{2 \pi t}{T}\right)\right] & (0<t<T) . \\
\alpha_{2} & (t \geq T)\end{cases}
\end{aligned}
$$

These are continuous up to the second derivatives, whereas the third derivatives are discontinuous at $t=0$ and $T$. Figure 1(a) shows the calculated results for the number of created polaritons of each mode of the lower branch, $\tilde{n}_{a 2}^{\lambda}(\mathbf{k})$, for various values of $\omega_{1} T$. Figure $1(\mathrm{~b})$ shows those of the upper branch, $\tilde{n}_{b 2}^{\lambda}(\mathbf{k})$. In both cases, strong excitonic features appear at $c k / \omega_{1} \simeq 1$ (which also means $c k / \omega_{2} \simeq 1$ because we have taken $\omega_{1} \simeq \omega_{2}$ ). When $c k<\omega_{i}$ $\left(c k>\omega_{i}\right)$, UB (LB) polaritons are created more efficiently than LB (UB) polaritons. This crossover occurs because we are here varying the exciton parameters, and the UB polariton has more (less) exciton component for $c k<\omega_{i}\left(c k>\omega_{i}\right)$. These characteristics are correctly described only by a microscopic model.

We also find that $\tilde{n}_{a 2}^{\lambda}$ decreases very quickly as $T$ is increased. To understand the decrease, let us investigate the case when $k=20 \omega_{1} / c$ and $\alpha_{1}=\alpha_{2}=0.001 \omega_{1} \sqrt{\epsilon_{0} \rho}$. For such large $k$ and small $\alpha$, a LB polariton is almost an exciton, and we can understand the physics clearly. The dotted line of Fig. 2 plots $\tilde{n}_{a 2}^{\lambda}$ for this case as a function of $\omega_{1} T$. We find that $\tilde{n}_{a 2}^{\lambda}$ decreases exponentially as $T$ is increased. We also find small oscillations for large $T$. This is found to be due to the fact that our $\omega_{x}(t)$ has singularities (third-order derivative is discontinuous at $t=0$ and $T$ ). To verify this we also consider the case when $\omega_{x}(t)$ takes the following analytic form [四;

$$
\omega_{x}^{2}(t)=\omega_{1}^{2}+\frac{\left(\omega_{2}^{2}-\omega_{1}^{2}\right)}{1+\exp (-t / \tau)},
$$

where $T=10 \tau$. (The coefficient is chosen in such a way that a small-T behavior agrees with the case of the non-analytic $\omega_{x}$.) To simplify discussion, let us take $\alpha=0$, for which a LB polariton becomes a pure exciton, and $\tilde{n}_{a 2}^{\lambda}$ becomes independent of $\mathbf{k}$. We then obtain the exact solution:

$$
\tilde{n}_{a 2}^{\lambda}=\frac{\sinh ^{2}\left[\pi\left(\omega_{1}-\omega_{2}\right) \tau\right]}{\sinh \left[2 \pi \omega_{1} \tau\right] \sinh \left[2 \pi \omega_{2} \tau\right]} .
$$

This is plotted in Fig. 2 with solid line. It is seen that the non-analyticity of the form (17) induces oscillations. It was also shown [4] that for large $\omega_{1} T(>20)$ the non-analyticity enhances $\tilde{n}_{a 2}^{\lambda}$ by many orders of magnitude. Since non-analytic change of $\omega_{x}(t)$ is unphysical, the result of analytic change of $\omega_{x}(t)$ should be more convincing. However, most previous studies [10, 11, 12] assumed that a time-dependent parameter (such as the dielectric constant) itself has discontinuities. Our results strongly suggest that such strong singularities would lead to physically incorrect results. Regarding our results of Fig. 1, they may be convincing because our slight singularity does not play important roles for such short $T$, as seen from Fig. 2.

To summarize this section, we have considered a polariton system in which excitonic parameters are time-dependent. The time dependence causes creation of polaritons from a "false vacuum," and we have evaluated the number of created polaritons for each polari- 
important, and the dielectric constant exhibits strong dispersions, we have employed a microscopic model in which the polarization degrees of freedom are included as microscopic variables. Moreover, whereas most previous studies assumed sudden changes $(T=0)$ of a parameter(s), we have assumed more realistic situations in which excitonic parameters vary within a finite time $(T>0)$. Our results strikingly differ from the previous results, both qualitatively (strong excitonic features at $c k / \omega_{1} \simeq 1$ ) and quantitatively (by many orders of magnitude).

\section{Polaritons in absorptive and inhomogeneous cavities}

It is usually assumed in discussions of polaritons that the excitation level, which couples to photons, of the material is a discrete level. The excitation level may be an exciton level in the case of an excitonic polariton, or a phonon level for a phonon polariton. As the photon energy is increased to a continuous absorption spectrum, the single-level approximation breaks down. Even in such a case, however, we may treat, to a first approximation, the material excitations as bosons (polarization fields) because the excitations are composed of bosons or pair excitations of fermions. We may therefore write the Lagrangian density, assuming a one-dimensional system for simplicity, as [7]

$$
\begin{aligned}
\mathcal{L} & =\mathcal{L}_{E M}+\mathcal{L}_{\text {mat }}+\mathcal{L}_{\text {int }} \\
\mathcal{L}_{E M} & =\frac{\epsilon_{0}}{2}\left[\dot{A}^{2}-c^{2}\left(\frac{\partial A}{\partial x}\right)^{2}\right] \\
\mathcal{L}_{\text {mat }} & =\frac{1}{2} \int_{0}^{\infty} d \omega \tilde{\rho}_{\omega}\left(\dot{\tilde{X}}_{\omega}^{2}-\omega^{2} \tilde{X}_{\omega}^{2}\right), \\
\mathcal{L}_{\text {int }} & =-\int_{0}^{\infty} d \omega \tilde{\alpha}_{\omega} A \dot{\tilde{X}}_{\omega}
\end{aligned}
$$

where $A(x, t)$ is the vector potential of the electromagnetic (EM) field, and $\tilde{X}_{\omega}(x, t)$ denotes the polarization field of frequency $\omega$. Since we are considering the case when the photon energy lies in a continuous absorption spectrum, we have taken $\tilde{X}_{\omega}(x, t)$ to have a continuous label $\omega$, and integral over $\omega$ is performed in Eqs. (23) and (24). Moreover, we are interested in the case when the spatial distribution of the material is inhomogeneous because in such a case the material constitutes a lossy cavity, in which behavior of polaritons should be very interesting. When the material exists only in the regions of $|x| \geq \ell / 2$, we may express this inhomogeneous distribution by imposing

$$
\tilde{X}_{\omega}(x, t)=0 \text { for }|x|<\ell / 2 .
$$

The equal-time canonical quantization of $A$ and $\tilde{X}_{\omega}$ can be performed in the standard manner, and we have found that the Hamiltonian is diagonalized as [7]

$$
H=\sum_{\sigma= \pm} \sum_{q} \int d \omega \hbar \omega a^{(q \sigma)}(\omega)^{\dagger} a^{(q \sigma)}(\omega)
$$

Here, $a^{(q \sigma)}(\omega)$ is the annihilation operator of a polariton, which is given by

$$
a^{(q \sigma)}(\omega) \equiv \sum_{j}\left(\beta_{j}^{(q \sigma)}(\omega) E_{j \sigma}+\tilde{\beta}_{j}^{(q \sigma)}(\omega) A_{j \sigma}\right)
$$


where $\sigma= \pm 1$ and $j=0,1,2, \cdots$ (or, $\sigma$ and $q=1,2, \cdots$ ) label some modes of the cavity, $-\epsilon_{0} E$ and $P$ are conjugate momenta of $A$ and $X$, respectively, and $\beta, \tilde{\beta}, \gamma$ and $\tilde{\gamma}$ are some coefficients. (For details of the notations, see Ref. [7].) It is seen that $a^{(q \sigma)}(\omega)$ is a superposition of infinite number of modes (of $A$ and $X$ ). In particular, the integration over continuous exciton modes results in the breakdown of the standard picture, that a polariton is a back and forth oscillation of photon $\rightarrow$ exciton $\rightarrow$ photon $\rightarrow$ exciton $\rightarrow \cdots$. That is, when we prepare a photon state as an initial state, the state will evolve into a superposition of infinite number of exciton modes, and never returns to the initial photon state.

Using the solution of such unusual polaritons, we can investigate various physical phenomena in absorptive and inhomogeneous cavities. For example, we have evaluated the radiative lifetime of an excited atom in such a cavity [7]. Calculations of other quantities are in progress.

\section{Acknowledgment}

This work has been supported by the Core Research for Evolutional Science and Technology (CREST) of the Japan Science and Technology Corporation (JST), and by Grants-in-Aid for Scientific Research on Priority Areas from the Ministry of Education, Science and Culture, and by Sumitomo Foundation.

\section{References}

[1] For a review, see, e.g., R. Matloob, R. Loudon, S.M. Barnett and J. Jeffers, Phys. Rev. A54 (1995) 4823.

[2] J.J. Hopfield, Phys. Rev. 112 (1958) 1555.

[3] H. Huttner, J.J. Baumberg and S.M. Barnett, Phys. Rev. A46 (1992) 4306.

[4] T. Okushima and A. Shimizu, Jpn. J. Appl. Phys. 34 (1995) 4508-4510.

[5] For a review, see, e.g., S. A. Fulling, Aspects of Quantum Field Theory in Curved Space-Time (Cambridge, 1989).

[6] E. Sassaroli, Y. N. Srivastava and A. Widom, Phys. Rev. A50 (1994) 1027.

[7] K. Koshino and A. Shimizu, Phys. Rev. A53 (1996) 4468.

[8] Note that in our problem the Fock space for $t<0$ is different from that for $t>T$ because the parameters which are used to define the Fock spaces are different. Hence, the equivalence of the Scrödinger picture and Heisenberg picture does not necessarily hold (if the system size is infinite). Although we explain the physics using the Shrödinger picture, the Heisenberg picture is more appropriate to treat this problem. Actual calculations have been performed in the Heisenberg picture.

[9] See, e.g., A. Yariv, Quantum Electronics, 3rd ed. (Wiley, NewYork,1989), section 17.7.

[10] E. Yablonovitch, Phys. Rev. Lett. 62 (1989) 1742.

[11] J. Schwinger, Proc. Natl. Acad. Sci. USA 90 (1993) 958-959. 
[12] Reference [11] introduced a finite time scale $\tau$ which in a sense characterizes time dependence of the dielectric constant. However, instantaneous changes were assumed at $t= \pm \tau / 2$, which seems to be more relevant in determining the number of created photons. The same can be said about the model shown in Fig. 5 of Ref. [6].

\section{Figure captions}

Fig. 1. The number of created polaritons per mode for (a) lower- and (b) upper-branch polaritons. The polariton parameters are taken as $\omega_{2}=0.95 \omega_{1}, \alpha_{1}=0.05 \omega_{1} \sqrt{\epsilon_{0} \rho}, \alpha_{2}=0.03 \omega_{1} \sqrt{\epsilon_{0} \rho}$, and $\omega_{1} T=0.1,2,4$.

Fig. 2. The number of created polaritons per mode for lower-branch (LB) polaritons is plotted as a function of $\omega_{1} T$. Dotted line is the result for the LB polariton of $k=20 \omega_{1} / c$, for $\alpha_{1}=\alpha_{2}=$ $0.001 \omega_{1} \sqrt{\epsilon_{0} \rho}$. Thin line represents the result when $\omega_{x}(t)$ takes the analytic form, Eq. (19), and $\alpha=0$ (which means that a LB polariton in this case is a pure exciton). In both cases $\omega_{2}$ is taken to be $0.9 \omega_{1}$. 
This figure "NEC_fig1a.jpg" is available in "jpg" format from: http://arxiv.org/ps/quant-ph/9804028v1 
This figure "NEC_fig1b.jpg" is available in "jpg" format from: http://arxiv.org/ps/quant-ph/9804028v1 
This figure "NEC_fig2.jpg" is available in "jpg" format from: http://arxiv.org/ps/quant-ph/9804028v1 